\title{
ON THE ELASTOPLASTIC STABILITY OF A PLATE UNDER SHEAR FORCES, TAKING INTO ACCOUNT ITS REAL BENDING FORM
}

\author{
DAO HUY BICH \\ Vietnam National University, Hanoi
}

\begin{abstract}
Using the theory of elastoplastic processes and the modified elastic solution method we investigate the stability outside elastic limit problem of a plate under shear forces, taking into account its real bending form after the loss of stability. An expression for determining the critical force is obtained and numerical calculations with various ratio of thickness have been fulfilled, from the results one can see the convergence of the modified elastic solution method.
\end{abstract}

\section{Introduction}

From the experimental results one can see that the bending form of the plate under shear forces after the loss of stability has a form of somewhat plane sloping roofs, the nodes of which are nearly straight lines. In the investigated case of a long elastic plate under shear forces, the slope makes with the long edge an angle about $54^{\circ}$ and the distance between the slopes is equal to 1.22 times of the plate width.

In the case of elastoplastic stability of a plate subjected to axial and shear forces, considered in [1], because of the complicatedness the author proposes some assumptions about the bending form. For reflecting the reality the real bending form must be taken into consideration of the elastoplastic stability of plates under shear forces.

\section{Formulation of the problem and the solving method}

Suppose a long plate of width $b$ subjected to the shear forces $\tau$ along its long edges $y=0$ and $y=b$ (see Fig. 1). At any moment $t$ there exists a membrane plane stress in the plate

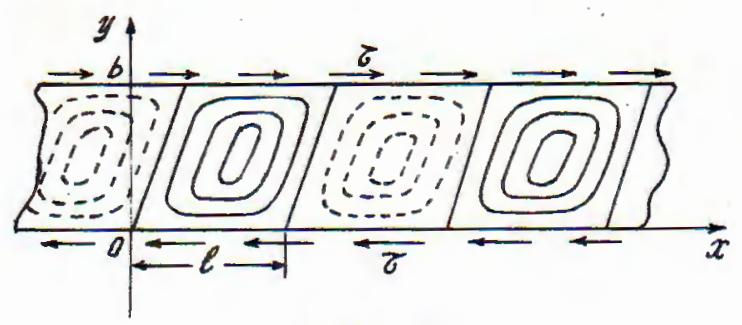

Fig. 1 


$$
\sigma_{11}=0, \quad \sigma_{22}=0, \quad \sigma_{12}=\tau, \quad \text { so that } \quad \sigma_{u}=\sqrt{3} \tau \text {. }
$$

Respectively, the strain state is determined by the stress-strain relationship of the elastoplastic process theory [1], in this case it has of the form

$$
\begin{aligned}
& \dot{\varepsilon}_{11}=0, \quad \dot{\varepsilon}_{22}=0 \\
& \dot{\varepsilon}_{12}=\frac{3 \dot{\tau}}{2 A}+\frac{3}{2}\left(\frac{1}{P}-\frac{1}{A}\right) \frac{3 \tau \dot{\tau}}{\sigma_{u}^{2}} \tau=\frac{3}{2} \frac{\dot{\tau}}{P}
\end{aligned}
$$

where $A=\frac{\sigma_{u}}{s}, P=\phi^{\prime}(s), s$-the arc-length of the strain trajectory:

$$
\frac{d s}{d t}=\frac{2}{\sqrt{3}}\left(\dot{\varepsilon}_{11}^{2}+\dot{\varepsilon}_{22}^{2}+\dot{\varepsilon}_{11} \dot{\varepsilon}_{22}+\dot{\varepsilon}_{12}^{2}\right)^{1 / 2}=\frac{2}{\sqrt{3}} \dot{\varepsilon}_{12},
$$

From $(2.1) \div(2.3)$ we have

$$
\frac{3}{2 \phi^{\prime}(s)} \frac{d \tau}{d t}=\frac{\sqrt{3}}{2} \frac{d s}{d t}, \quad \text { or } \quad \sqrt{3} d \tau=\phi^{\prime}(s) d s,
$$

that yields

$$
\sqrt{3} \tau=\sigma_{u}=\phi(s)
$$

At the moment an instability occurs, a bifurcation of equilibrium states is assumed to appear, with an infinitesimal small increment of the external force there are possible increments of deformations (including the bending deformation). Respectively, the internal bending moments [1] in this case are determined through the deflection $w$ of the plate as follows

$$
\begin{aligned}
& M_{11}=-\frac{A h^{3}}{18}\left(2 \frac{\partial^{2} w}{\partial x^{2}}+\frac{\partial^{2} w}{\partial y^{2}}\right) \\
& M_{22}=-\frac{A h^{3}}{18}\left(\frac{\partial^{2} w}{\partial x^{2}}+2 \frac{\partial^{2} w}{\partial y^{2}}\right) \\
& M_{12}=-\frac{A h^{3}}{18}\left(\frac{\partial^{2} w}{\partial x \partial y}+\left(\frac{P}{A}-1\right) \frac{\partial^{2} w}{\partial x \partial y}\right) .
\end{aligned}
$$

The bending energy of the plate now is represented as

$$
\begin{gathered}
\delta U=-\iint\left[M_{11} \delta\left(\frac{\partial^{2} w}{\partial x^{2}}\right)+M_{22} \delta\left(\frac{\partial^{2} w}{\partial y^{2}}\right)+2 M_{12} \delta\left(\frac{\partial^{2} w}{\partial x \partial y}\right)\right] d x d y \\
=\frac{A h^{3}}{18} \delta \iint\left[\left(\frac{\partial^{2} w}{\partial x^{2}}+\frac{\partial^{2} w}{\partial y^{2}}\right)^{2}+\left(\frac{\partial^{2} w}{\partial x \partial y}\right)^{2}-\frac{\partial^{2} w}{\partial x^{2}} \frac{\partial^{2} w}{\partial y^{2}}\right. \\
\left.+\left(\frac{P}{A}-1\right)\left(\frac{\partial^{2} w}{\partial x \partial y}\right)_{1}^{2}\right] d x d y
\end{gathered}
$$


owing to $A, P$ not depending on $w$. On the other hand we demonstrate the work of shear forces

$$
L=\iint \tau h \frac{\partial w}{\partial x} \frac{\partial w}{\partial y} d x d y
$$

Experimental results show that when the instability occurs, the plate makes up bending slopes, the nodes of which are nearly straight lines, therefore we choose the deflection of a plate in the form

$$
w=f \sin \frac{\pi y}{b} \sin \frac{\pi}{\ell}(x-\gamma y) .
$$

This deflection will be equal to zero at the long edges and at the node lines $x-\gamma y=$ $k \ell$, where $k=0, \pm 1, \pm 2, \ldots ; \ell$ is the distance between the nodes; $\gamma, \ell$ - unknown quantities to be determined.

The elastoplastic stability problem can be solved by using the energy method. For this aim, it is necessary to evaluate the bending energy of the plate and the work of shear forces. Substituting (2.7) into (2.5), (2.6)

$$
\begin{aligned}
U= & \frac{A h^{3}}{18} \int_{0}^{b} \int_{x-\gamma y=k \ell}^{x-\gamma y=(k+1) \ell}\left\{\left[-f \pi^{2}\left(\frac{1}{\ell^{2}}+\frac{\gamma^{2}}{\ell^{2}}+\frac{1}{b^{2}}\right) \sin \frac{\pi y}{b} \sin \frac{\pi}{\ell}(x-\gamma y)\right.\right. \\
& \left.-2 f \frac{\pi^{2} \gamma}{b \ell} \cos \frac{\pi y}{b} \cos \frac{\pi}{\ell}(x-\gamma y)\right]^{2}+\left[f \frac{\pi^{2} \gamma}{\ell^{2}} \sin \frac{\pi y}{b} \sin \frac{\pi}{\ell}(x-\gamma y)\right. \\
& \left.+f \frac{\pi^{2}}{b \ell} \cos \frac{\pi y}{b} \cos \frac{\pi}{\ell}(x-\gamma y)\right]^{2}-f \frac{\pi^{2}}{\ell^{2}} \sin \frac{\pi y}{b} \sin \frac{\pi}{\ell}(x-\gamma y) \times \\
& \times\left[f\left(\frac{\pi^{2} \gamma^{2}}{\ell^{2}}+\frac{\pi^{2}}{b^{2}}\right) \sin \frac{\pi y}{b} \sin \frac{\pi}{\ell}(x-\gamma y)+2 f \frac{\pi^{2} \gamma}{b \ell} \cos \frac{\pi y}{b} \cos \frac{\pi}{\ell}(x-\gamma y)\right] \\
& \left.+\left(\frac{P}{A}-1\right)\left[f \frac{\pi^{2} \gamma}{\ell^{2}} \sin \frac{\pi y}{b} \sin \frac{\pi}{\ell}(x-\gamma y)+f \frac{\pi^{2}}{b \ell} \cos \frac{\pi y}{b} \cos \frac{\pi}{\ell}(x-\gamma y)\right]^{2}\right\} d x d y, \\
L= & \int_{0}^{b-\gamma y=(k+1) \ell} \int_{x-\gamma y=k \ell} \int^{2}\left(\frac{\pi f^{2}}{\ell^{2}} \sin ^{2} \frac{\pi y}{b} \cos ^{2} \frac{\pi}{\ell}(x-\gamma y)\right. \\
& \left.-\frac{\pi^{2}}{b \ell} \sin \frac{\pi y}{b} \cos \frac{\pi y}{b} \sin \frac{\pi}{\ell}(x-\gamma y) \cos \frac{\pi}{\ell}(x-\gamma y)\right] d x d y \quad k=0, \pm 1, \pm 2, \ldots
\end{aligned}
$$

after some operations we receive

$$
\begin{aligned}
U & =\frac{A h^{3} f^{2} \pi^{4}}{72 b \ell}\left[2+6 \gamma^{2}+\left(\frac{\ell}{b}\right)^{2}+\left(\frac{b}{\ell}\right)^{2}\left(1+\gamma^{2}\right)^{2}+\left(\frac{P}{A}-1\right)\left(1+\gamma^{2} \frac{b^{2}}{\ell^{2}}\right)\right], \\
L & =\frac{\tau f^{2} \pi^{2} \gamma b h}{4 \ell} .
\end{aligned}
$$


Equating expressions of the bending energy and the work of shear forces gives the relation for finding critical forces

$$
\tau=\frac{\pi^{2} A h^{2}}{18 \gamma b^{2}}\left[6 \gamma^{2}+\left(\frac{\ell}{b}\right)^{2}+\left(\frac{b}{\ell}\right)^{2}\left(1+\gamma^{2}\right)^{2}+2+\left(\frac{P}{A}-1\right)\left(1+\gamma^{2}\left(\frac{b}{\ell}\right)^{2}\right)\right] .
$$

Further one needs to seek a minimum of $\tau$. Consider two cases:

\section{a. The case of an elastic plate}

In this case $A=P=3 G$ the expression (2.10) becomes

$$
\tau=\frac{\pi^{2} D}{2 \gamma h b^{2}}\left[6 \gamma^{2}+2+\left(\frac{\ell}{b}\right)^{2}+\left(\frac{b}{\ell}\right)^{2}\left(1+\gamma^{2}\right)^{2}\right]
$$

which entirely coincides with the result of an elastic plate made of an incompressible material under shear forces [3] with

$$
D=\frac{G h^{3}}{3}=\frac{E h^{3}}{9}
$$

Putting $\xi=\frac{\ell}{b}$ into (2.11)

$$
\tau=\frac{\pi^{2} D}{2 \gamma h b^{2}}\left[6 \gamma^{2}+2+\xi^{2}+\frac{1}{\xi^{2}}\left(1+\gamma^{2}\right)^{2}\right]
$$

then minimizing the obtained expression

$$
\frac{\partial \tau}{\partial \xi}=0, \quad \frac{\partial \tau}{\partial \gamma}=0
$$

gives us

$$
\begin{aligned}
& \xi^{2}=1+\gamma^{2} \\
& 2+\xi^{2}+\frac{1+\gamma^{2}}{\xi^{2}}\left(1-3 \gamma^{2}\right)-6 \gamma^{2}=0
\end{aligned}
$$

from where it follows $\gamma=\frac{1}{\sqrt{2}}, \xi=1.22$ or $\ell=1.22 b$.

The critical forces have been determined

$$
\tau_{c r}^{(e)}=4 \sqrt{2} \frac{\pi^{2} D}{b^{2} h}
$$

\section{b. The case of an elastoplastic plate}

In this case 


$$
\begin{aligned}
& P=\phi^{\prime}(s)=E_{t}(s), \\
& A=\frac{\sigma_{u}}{s}=\frac{\sqrt{3} \tau}{s}=E_{c}(s),
\end{aligned}
$$

where $E_{t}(s)$ - the tangential modulus, $E_{c}(s)$ - the secant modulus of the material and with denotation $i^{2}=\frac{9 b^{2}}{h^{2}}$, the equation (2.10) becomes

$$
i^{2}=\frac{\pi^{2} \sqrt{3}}{2 \gamma s}\left[2+6 \gamma^{2}+\xi^{2}+\frac{1}{\xi^{2}}\left(1+\gamma^{2}\right)^{2}+\left(\frac{E_{t}(s)}{E_{c}(s)}-1\right)\left(1+\frac{\gamma^{2}}{\xi^{2}}\right)\right] .
$$

Minimizing $i^{2}$

$$
\frac{\partial i^{2}}{\partial \xi}=0, \quad \frac{\partial i^{2}}{\partial \gamma}=0
$$

that, yields

$$
\begin{aligned}
& 1-\frac{\left(1+\gamma^{2}\right)^{2}}{\xi^{4}}-\left(\frac{E_{t}(s)}{E_{c}(s)}-1\right) \frac{\gamma^{2}}{\xi^{4}}=0 \\
& 2+\xi^{2}+\frac{1+\gamma^{2}}{\xi^{2}}\left(1-3 \gamma^{2}\right)-6 \gamma^{2}+\left(\frac{E_{t}(s)}{E_{c}(s)}-1\right)\left(1-\frac{\gamma^{2}}{\xi^{2}}\right)=0 .
\end{aligned}
$$

The expression (2.13) can be rewritten in the other form

$$
s=\frac{\pi^{2} \sqrt{3}}{2 \gamma i^{2}}\left[2+6 \gamma^{2}+\xi^{2}+\frac{1}{\xi^{2}}\left(1+\gamma^{2}\right)^{2}+\left(\frac{E_{t}(s)}{E_{c}(s)}-1\right)\left(1+\frac{\gamma^{2}}{\xi^{2}}\right)\right] .
$$

The problem determining critical forces of a plate under shear forces reduces to seek $\gamma, \xi$ from the equations (2.14), (2.15), then a substitution of just obtained values into (2.16) gives the relation for finding the critical value $s_{c r}$. Finally the critical forces can be evaluated from

$$
\tau_{c r}=\frac{1}{\sqrt{3}} \phi\left(s_{c r}\right)
$$

Since the system of equations $(2.14) \div(2.16)$ is nonlinear, we solve this system by using the modified elastic solution method [2].

On the first iteration by putting $E_{t}(s)=E_{c}(s)=3 G$ from $(2.14),(2.15)$ we get again known values $\gamma_{1}=\frac{1}{\sqrt{2}}, \xi_{1}=1.22$ (respectively to the elastic case). Substitute them to $(2.16)$ for determining $s_{1}$

$$
s_{1}=\frac{\pi^{2} \sqrt{3}}{2 \gamma_{1} i^{2}}\left[2+6 \gamma_{1}^{2}+\xi_{1}^{2}+\frac{1}{\xi_{1}^{2}}\left(1+\gamma_{1}^{2}\right)^{2}\right] \text {. }
$$


If $s_{1} \leq \varepsilon_{s}$, the obtained result $\tau_{c r}^{(1)}=\frac{1}{\sqrt{3}} \phi\left(s_{1}\right)$ is an elastic solution. It is already an exact solution.

If $s_{1}>\varepsilon_{s}$ we remove to the serond iteration. A procedure of the iterative method for solving $(2.14) \div(2.16)$ can be written as following

$$
\begin{aligned}
& \xi_{n}^{2}=\left(1+\gamma_{n}^{2}\right)\left[1+\frac{\left(\frac{E_{t}\left(s_{n-1}\right)}{E_{c}\left(s_{n-1}\right)}-1\right) \gamma_{n-1}^{2}}{\left(1+\gamma_{n-1}^{2}\right)^{2}}\right]^{1 / 2}, \\
& 2+\xi_{n}^{2}-6 \gamma_{n}^{2}+\frac{1+\gamma_{n}^{2}}{\xi_{n}^{2}}\left(1-3 \gamma_{n}^{2}\right)+\left(\frac{E_{t}\left(s_{n-1}\right)}{E_{c}\left(s_{n-1}\right)}-1\right)\left(1-\frac{\gamma_{n-1}^{2}}{\xi_{n-1}^{2}}\right)=0, \\
& s_{n}=\frac{\pi^{2} \sqrt{3}}{2 \gamma_{n} i^{2}}\left[2+6 \gamma_{n}^{2}+\xi_{n}^{2}+\frac{1}{\xi_{n}^{2}}\left(1+\gamma_{n}^{2}\right)^{2}+\left(\frac{E_{t}\left(s_{n-1}\right)}{E_{c}\left(s_{n-1}\right)}-1\right)\left(1+\frac{\gamma_{n-1}^{2}}{\xi_{n-1}^{2}}\right)\right]
\end{aligned}
$$

and the critical force is determined by

$$
\tau_{c r}^{(n)}=\frac{1}{\sqrt{3}} \phi\left(s_{n}\right) .
$$

All values $\gamma_{n-1}, \xi_{n-1}, s_{n-1}$ are considered to be known at $(n-1)$-th iteration, from (2.17), (2.18) $\gamma_{n}, \xi_{n}$ are evaluated and then $s_{n}, \tau_{c r}^{(n)}$ - from (2.19), (2.20). Denoting

$$
\begin{aligned}
& \alpha_{n-1}=\left[1+\frac{\left(\frac{E_{t}\left(s_{n-1}\right)}{E_{c}\left(s_{n-1}\right)}-1\right) \gamma_{n-1}^{2}}{\left(1+\gamma_{n-1}^{2}\right)^{2}}\right]^{1 / 2}, \\
& \beta_{n-1}=\left(\frac{E_{t}\left(s_{n-1}\right)}{E_{c}\left(s_{n-1}\right)}-1\right)\left(1-\frac{\gamma_{n-1}^{2}}{\xi_{n-1}^{2}}\right), \\
& \delta_{n-1}=\left(\frac{E_{t}\left(s_{n-1}\right)}{E_{c}\left(s_{n-1}\right)}-1\right)\left(1+\frac{\gamma_{n-1}^{2}}{\xi_{n-1}^{2}}\right),
\end{aligned}
$$

from $(2.17) \div(2.20)$ by means of some straightforward operations the system of equations for $n$-th iteration is obtained

$$
\begin{aligned}
\gamma_{n}^{2} & =\frac{2+\alpha_{n-1}+\frac{1}{\alpha_{n-1}}+\beta_{n-1}}{6+\frac{3}{\alpha_{n-1}}-\alpha_{n-1}}, \\
\xi_{n}^{2} & =\alpha_{n-1}\left(1+\gamma_{n}^{2}\right), \\
s_{n} & =\frac{\pi^{2} \sqrt{3}}{2 \gamma_{n} i^{2}}\left[2+6 \gamma_{n}^{2}+\xi_{n}^{2}+\frac{1}{\xi_{n}^{2}}\left(1+\gamma_{n}^{2}\right)^{2}+\delta_{n-1}\right], \\
\tau_{c r}^{(n)} & =\frac{1}{\sqrt{3}} \phi\left(s_{n}\right) .
\end{aligned}
$$


The solving procedure consists of the following steps: firstly $\gamma_{n}$ is determined from (2.21), this value is then substituted into (2.21), from where $\xi_{n}$ is obtained, further by putting just results $\gamma_{n}, \xi_{n}$ into (2.23) we get $s_{n}$ and then from $(2.24)-\tau_{c r}^{(n)}$

Practically, the iterative process will be finished when

$$
\left|\frac{s_{n}-s_{n-1}}{s_{n-1}}\right|<\varepsilon,
$$

where $\varepsilon$ is a given forward small value.

\section{Elastoplasic stability problem of a plate of comparable sides}

Let's consider a plate of comparable sides $a$ and $b$, subjected to shear forces and simply supported on its edges. When the instability occurs, the bending form of the plate depends much more on the ratio of its sides. Because of the difficulty to predict the real bending form, then by using the Ritz method an approximate solution is chosen

$$
w=\sum_{m=1}^{\infty} \sum_{n=1}^{\infty} a_{m n} \sin \frac{m \pi x}{a} \sin \frac{n \pi y}{b}
$$

The bending energy is evaluated by the formula (2.5)

$$
U=\frac{\pi^{4} a b A h^{3}}{72} \sum_{m} \sum_{n} a_{m n}^{2}\left[\left(\frac{m^{2}}{a^{2}}+\frac{n^{2}}{b^{2}}\right)^{2}+\frac{m^{2} n^{2}}{a^{2} b^{2}}\left(\frac{P}{A}-1\right)\right] .
$$

For calculating the work of shear forces by (2.6), it needs to involve such integral

$$
\int_{0}^{a} \sin \frac{i \pi x}{a} \cos \frac{m \pi x}{a} d x=\left\{\begin{array}{cl}
\frac{2 a}{\pi} \frac{i}{i^{2}-m^{2}} & \text { with } i+m \text {-odd number } \\
0 & \text { with } i+m \text {-even number }
\end{array}\right.
$$

finally we obtain

$$
L=4 \tau h \sum_{m} \sum_{n} \sum_{i} \sum_{j} a_{m n} a_{i j} \frac{m n i j}{\left(i^{2}-m^{2}\right)\left(j^{2}-n^{2}\right)} .
$$

The expression (3.3) contains only terms with $m+i, n+j$ taking odd numbers.

The minimum condition of the total energy

$$
\frac{\partial \ni}{\partial a_{m n}}=\frac{\partial(U-L)}{\partial a_{m n}}
$$


gives us

$$
\begin{aligned}
& \frac{\pi^{2} a b A h^{3}}{36} a_{m n}\left[\left(\frac{m^{2}}{a^{2}}+\frac{n^{2}}{b^{2}}\right)^{2}+\frac{m^{2} n^{2}}{a^{2} b^{2}}\left(\frac{P}{A}-1\right)\right] \\
& -8 \tau h \sum_{i} \sum_{j} a_{i j} \frac{m n i j}{\left(i^{2}-m^{2}\right)\left(j^{2}-n^{2}\right)}=0 \quad(m+i, n+j \text {-odd numbers }) .
\end{aligned}
$$

Solution to this system of equations is very complicated, then on the first approximation we choose

$$
m, n, i, j=\left\{\begin{array}{l}
1,1,2,2 \\
2,2,1,1 .
\end{array}\right.
$$

The system (3.4) reduces to

$$
\begin{aligned}
& \frac{\pi^{4} a b A h^{3}}{32}\left[\left(\frac{1}{a^{2}}+\frac{1}{b^{2}}\right)^{2}+\frac{1}{a^{2} b^{2}}\left(\frac{P}{A}-1\right)\right] a_{11}-4 \tau h a_{22}=0, \\
& 4 \tau h a_{11}-\frac{\pi^{4} a b A h^{3}}{2}\left[\left(\frac{1}{a^{2}}+\frac{1}{b^{2}}\right)^{2}+\frac{1}{a^{2} b^{2}}\left(\frac{P}{A}-1\right)\right] a_{22}=0 .
\end{aligned}
$$

Since $a_{11}, a_{22}$ are not vanished, then the determinant of this system must be equal to zero, from that we have

$$
\tau=\frac{\pi^{4} A h^{2}\left[\left(1+\alpha^{2}\right)^{2}+\alpha^{2}\left(\frac{P}{A}-1\right)\right]}{32 \alpha^{3} b^{2}},
$$

where $\alpha=\frac{a}{b}$ - the ratio of plate sides.

In the case of an elastic plate $A=P=3 G$, from (3.5) we receive the well-known value of critical forces [3]

$$
\tau_{c r}^{(e)}=\frac{3 \pi^{4} G h^{2}}{32 \alpha^{3} b^{2}}\left(1+\alpha^{2}\right)^{2}=\frac{27 \pi^{4} G\left(1+\alpha^{2}\right)^{2}}{32 \alpha^{3} i^{2}},
$$

where $i^{2}=9 \frac{b^{2}}{h^{2}}$

In the elastoplastic plate, by putting $A=\frac{\sigma_{u}}{s}=\frac{\sqrt{3} \tau}{s}=E_{c}(s), P(s)=\phi^{\prime}(s)=$ $E_{t}(s)$, the relation (3.5) can be rewritten in the form

$$
s=\frac{9 \sqrt{3} \pi^{4}\left[\left(1+\alpha^{2}\right)^{2}+\alpha^{2}\left(\frac{E_{t}(s)}{E_{c}(s)}-1\right)\right]}{32 \alpha^{3} i^{2}} .
$$

The equation (3.7) for finding $s_{c r}$ is a nonlinear equation with respect to $s$, to which the modified elastic solution method is applied. 
On the first iteration we take $E_{t}(s)=E_{c}(s)=3 G$, then

$$
s_{c s}^{(1)}=\frac{9 \sqrt{3} \pi^{4}\left(1+\alpha^{2}\right)^{2}}{32 \alpha^{3} i^{2}} \text {. }
$$

If $s_{c r}^{(1)} \leq \varepsilon_{s}$, the iteration process is finished, we have an elastic solution. If $s_{c r}^{(1)}>\varepsilon_{s}$ we remove to the second iteration. The iterative procedure must be continued as following

$$
s_{c r}^{(n)}=\frac{9 \sqrt{3} \pi^{4}\left[\left(1+\alpha^{2}\right)^{2}+\alpha^{2}\left(\frac{E_{t}\left(s_{n-1}\right)}{E_{c}\left(s_{n-1}\right)}-1\right)\right]}{32 \alpha^{3} i^{2}},
$$

where $n$-number of iteration. Substituting obtained $s_{c r}^{(n)}$ into

$$
\tau_{c r}^{(n)}=\frac{1}{\sqrt{3}} \phi\left(s_{c r}^{(n)}\right)
$$

we get the value of critical shear forces.

\section{Numerical examples and discussion}

a. A numerical analysis is considered on the long plate made of steel 30X CCA and subjected to shear forces. The steel 30XГCA is a hardening material with an elastic modulus $3 G=2.6 \cdot 10^{5} \mathrm{MPa}$, an yield point $\sigma_{s}=400 \mathrm{MPa}$, a stress versus strain curve $\phi(s)$ given by a table. The ratio $i=3 \frac{b}{h}$ varies from 120 to 300 .

The problem is solved by the iterative method described by the system of equations $(2.21) \div(2.24)$. Obtained results on the first, second and third iterations are shown on the table 1 . The elastic and elastoplastic solutions with respect to the critical forces are denoted by the numbers 1 and 2 on the Fig. 2.

The distance between the node lines $\ell$ and the slope $\gamma$ can be taken for the elastic case $\ell=1.22 b, \gamma=0.709$ and for the elastoplastic case $\ell \approx 1.13 b, \gamma \approx 0.64$. That means in the elastoplastic case the distance between the node lines is more narrow then one in the elastic case, while the sloping angle is about $57^{\circ}$ in comparison with $54^{\circ}$ in the elastic case. The bending form of a plate is shown on Fig. 1.

$\boldsymbol{b}$. Consider another example for a plate made of steel 30XГCA with the ratio of its sides $\alpha=\frac{a}{b}=\frac{1}{2}$. The stiffness of the plate $i=3 \frac{b}{h}$ varies from 270 to 600 . Numerical results by solving (3.8) are represented on the table 2 and the elastic and elasto-plastic solutions with respect to the critical forces are denoted by the numbers 3 and 4 on the Fig. 2.

From obtained results of two considered problems it can be seen that the elastic and elastoplastic solutions are much closer to each other in the cases with greater ratio $3 \frac{b}{h}$ and highly differ from each other in the cases with smaller one. Obviously, 


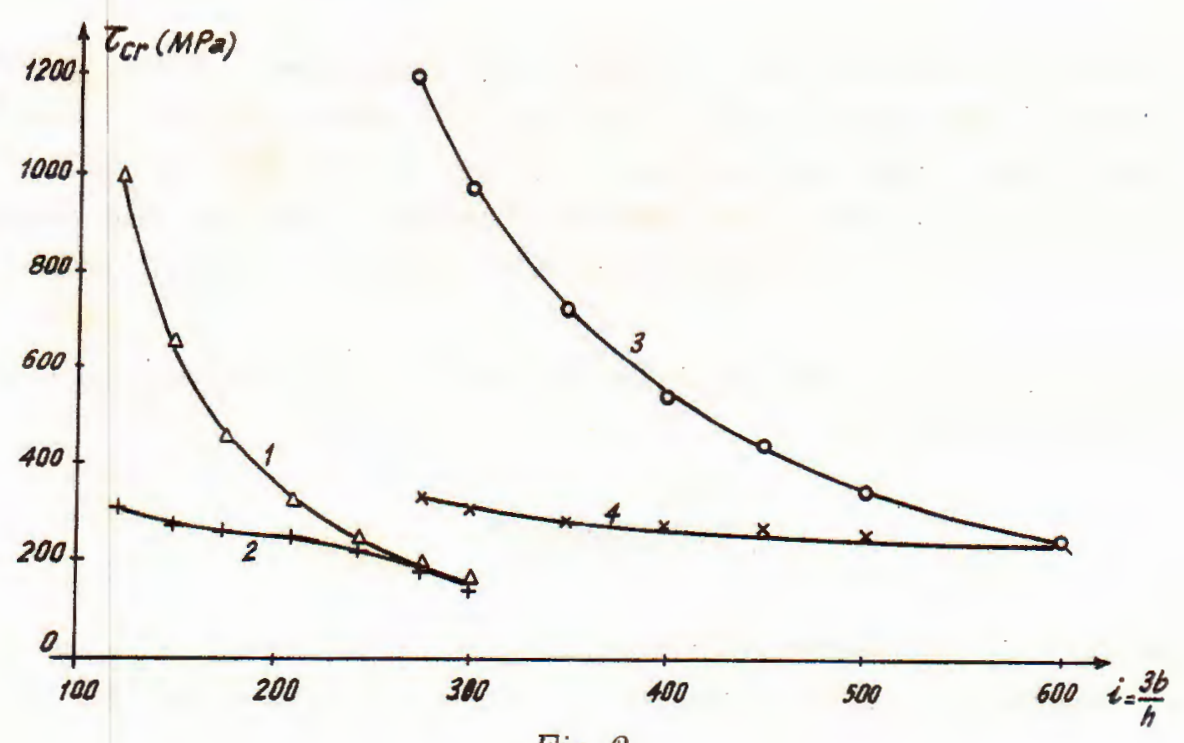

Fig. 2

Table 1

\begin{tabular}{cccccccccccccc}
\hline$i$ & $\begin{array}{c}\tau_{c r}^{(e)} \\
-\end{array}$ & $\begin{array}{c}\mathrm{MPa} \\
-\end{array}$ & $s_{1}$ & $\begin{array}{c}\tau_{c r}^{(1)} \\
\mathrm{MPa}\end{array}$ & $-\gamma_{2}$ & $\xi_{2}$ & $s_{2}$ & $\begin{array}{c}\tau_{c r}^{(2)} \\
\text { MPa }\end{array}$ & $\gamma_{3}$ & $\xi_{3}$ & $s_{3}$ & $\begin{array}{c}\tau_{c r}^{(3)} \\
\text { MPa }\end{array}$ \\
120 & 1004 & 0.006 & 306 & 0.64 & 1.127 & 0.0057 & 305 & 0.6398 & 1.131 & 0.0057 & 305 \\
150 & 642 & 0.0043 & 291 & 0.6394 & 1.1266 & 0.0036 & 286 & 0.6399 & 1.132 & 0.0036 & 286 \\
180 & 446 & 0.003 & 276 & 0,6387 & 1.1257 & 0.0025 & 269 & 0.6407 & 1.1329 & 0.0025 & 269 \\
210 & 327 & 0.0021 & 260 & 0.644 & 1.1339 & 0.0019 & 254 & 0.65 & 1.14 & 0.0019 & 254 \\
240 & 251 & 0.0016 & 242 & 0.6675 & 1.1675 & 0.00153 & 237 & 0.6748 & 1.172 & 0.00151 & 235 \\
270 & 198 & 0.0013 & 195 & 0.709 & 1.22 & & data coincide with elastic solution & \\
300 & 160 & 0.0010 & 158 & 0.709 & 1.22 & & data coincide with elastic solution & \\
\hline
\end{tabular}

Table 2

\begin{tabular}{|c|c|c|c|c|c|c|c|}
\hline$i$ & $\begin{array}{c}\tau_{c r}^{(e)} \\
\mathrm{MPa}\end{array}$ & $s_{1}$ & $\begin{array}{c}\tau_{c r}^{(1)} \\
\mathrm{MPa}\end{array}$ & $s_{2}$ & $\begin{array}{c}\tau_{c r}^{(2)} \\
\mathrm{MPa}\end{array}$ & $s_{3}$ & $\begin{array}{c}\tau_{c r}^{(3)} \\
\mathrm{MPa}\end{array}$ \\
\hline 270 & 1219 & 0.0080 & 318 & 0.0070 & 312 & 0.0070 & 312 \\
\hline 300 & 987 & 0.0062 & 307 & 0,0058 & 303 & 0.00575 & 303 \\
\hline 350 & 725 & 0.0048 & 295 & 0.0042 & 291 & 0.0042 & 291 \\
\hline 400 & 555 & 0.0036 & 284 & 0.0032 & 278 & 0.0032 & 278 \\
\hline 450 & 438 & 0.0029 & 276 & 0.0025 & 268 & 0.0025 & 268 \\
\hline 500 & 355 & 0.0023 & 262 & 0.0020 & 256 & 0.0020 & 256 \\
\hline 600 & 246 & 0.0016 & 236 & 0.0015 & 231 & 0.0015 & 231 \\
\hline
\end{tabular}


for plates with the same stiffness the instability happens in a long plate earlier then in a plate of comparable sides. The iterative process can be finished on the third iteration with a very high accuracy. These results also can talk about the convergence of the modified elastic solution method. Further, this method can be applied to considering elastoplastic stability problems of plates under complex loading.

This paper is completed with financial support from the National Basic Research Program in Natural Sciences.

\section{REFERENCE}

1. Dao Huy Bich. Elastoplastic stability of thin plates subjected to complex loading process. Journal of Mechanics, NCST of Vietnam T.XVI, 1994, No 1, pp. 2-8.

2. Dao Huy Bich. The modified method of elastic solution in the elasto plastic deformation process theory. Journal of Sciences, Hanoi University, series Math. and Phys., No 2, 1985, pp. 1-6, (in Vietnamese).

3. Volmir A. S. Stability of deformable systems. Edition of Sciences, Moscow 1967, (in Russian).

Received August 27, 2000

\section{VỀ SỰ ỔN ĐỊNH ĐÀN DÉO CƯA BẢN DƯỚT TÁC DỤNG CỦA LỰC TRỰ̛T CÓ TÍNH ĐẾN DẠNG VỒNG THỰC CƯA BẢN}

Sưr dụng lý thuyết quá trình đàn déo và phương pháp biến thể nghiệm đàn dèo để khảo sát bài toán ổn định ngoài giới hạn đàn hồi của bản dưới tác dụng của lực trượt có tính đến dạng vồng thực của nó sau khi mất ổn định. Đã nhận được biểu thi̛̛c xác định lực tới hạn. Đã thực hiện các tính toán bằng số với độ mảnh thay đổi, qua đó khẳng định sự hội tụ của phương pháp biến thể nghiệm đàn hồi. 